\title{
Towards SIMS on the Helium Ion Microscope: Detection Limits and Experimental Results on the ORION
}

\author{
D. Dowsett ${ }^{1}$, L. Pillatsch ${ }^{1}$, N.Vanhove ${ }^{1}$, T. Wirtz ${ }^{1}$, S. Sijbrandij ${ }^{2}$ and J. Notte ${ }^{2}$ \\ 1. Department "Science and Analysis of Materials" (SAM), Centre de Recherche Public - Gabriel \\ Lippmann, 41 rue du Brill, L-4422 Belvaux, Luxembourg \\ 2. Carl Zeiss Microscopy LLC, One Corporation Way, Peabody, MA, USA
}

The ORION Helium Ion Microscope has become a well established tool for high resolution microscopy [1] and nanofabrication [2]. The instrument is based on the atomic-sized ALIS gas field ion source, which has a brightness of $4 \times 10^{9} \mathrm{~A} \mathrm{~cm}^{-2} \mathrm{sr}^{-1}$ and which leads to a probe size of less than $0.5 \mathrm{~nm}$. The source can operate with both helium and neon [3].

While secondary electrons are used for high resolution imaging, some compositional information can be obtained from backscattered $\mathrm{He} / \mathrm{Ne}$ ions. In order to get chemical information with much higher sensitivity, we have previously investigated the feasibility of performing Secondary Ion Mass Spectrometry on the Helium Ion Microscope [4].

Our earlier studies concentrated first on potential useful yields, detection limits and achievable resolution and then on the practicality of secondary ion collection [5]. We have determined experimentally secondary ion yields under helium and neon bombardment for a range of semiconductor and metal samples. High secondary ion yields are crucial when using a small low current analytical probe. While basic yields are low due to the use of noble gas primary ions, they may be enhanced by several orders of magnitude for both negative and positive secondary ions by caesium and oxygen flooding respectively. Measurement of yields has allowed us to calculate detection limits for these samples under typical ORION imaging conditions (see figure 1).

More recently an extraction and detection system for secondary ions has been developed for the Helium Ion Microscope by the CRP - Gabriel Lippmann. We have investigated secondary ion emission for both semiconductor ( $\mathrm{Si}, \mathrm{InP}$ and $\mathrm{GaAs})$ and metal $(\mathrm{Cu}, \mathrm{Ni})$ samples on the ORION. Secondary to primary ion current ratios have been measured under He bombardment on the ORION and are consistent with those determined previously. Both total secondary ion depth profiles and secondary ion images (see figure 2) have been obtained under helium and neon bombardment.

The obtained results are very encouraging and the prospects of performing SIMS on the ORION are very interesting. By combing high resolution imaging with high sensitivity chemical mapping new nanoanalytical experiments will become possible leading to enhanced information about analyzed samples. 


\section{References:}

[1] L. Scipioni et al, J. Vac. Sci. Technol. B 27, 3250 (2009)

[2] D. Winston et al, Nano Letters 114343 (2011)

[3] F. Rahman et al., Scanning 33 (2011) 1

[4] T. Wirtz et al, Appl. Phys. Lett. 101041601 (2012)

[5] D. Dowsett et al, J. Vac. Sci. Technol. B 30 06F602 (2012)

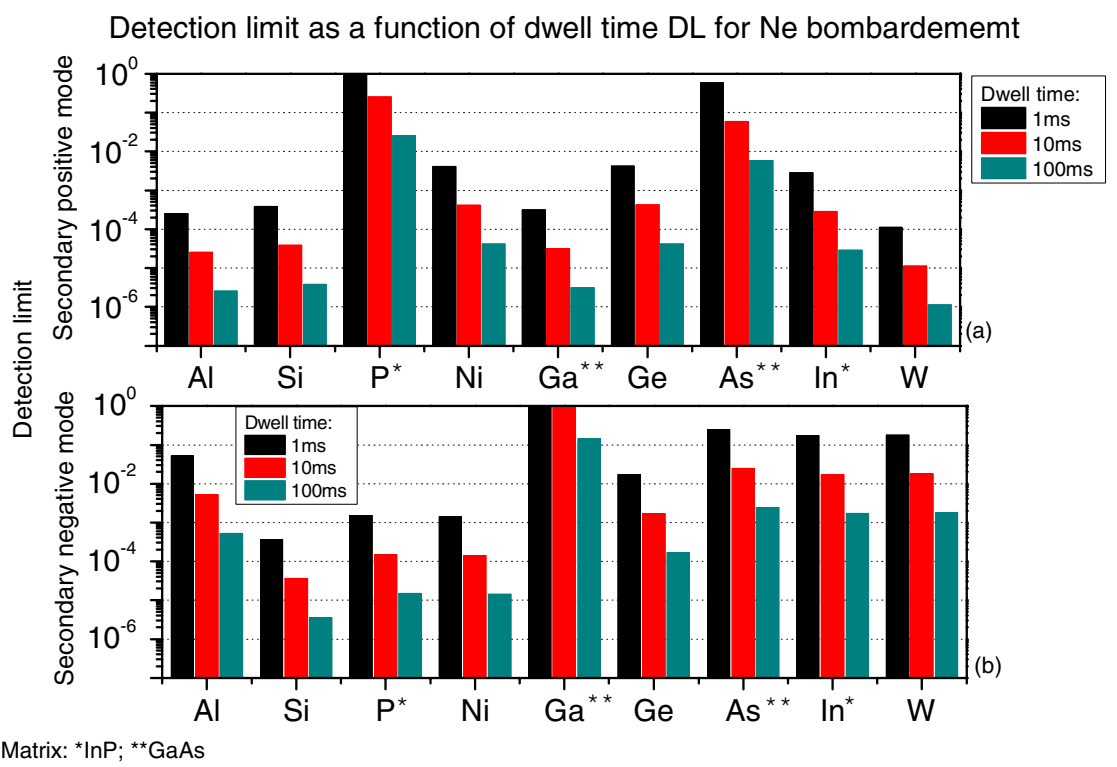

Figure 1. Detection limits for various semiconductor and metal samples under neon bombardment a) secondary positive mode with oxygen flooding b) secondary negative mode with caesium flooding.

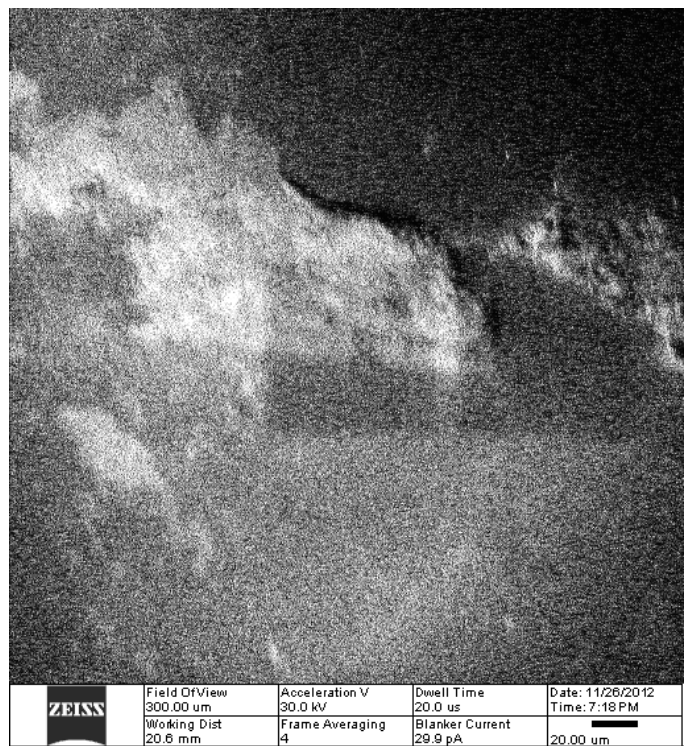

Figure 2. Total secondary ion image of the native oxide of nickel. Primary He current $30 \mathrm{pA}$, field of view $300 \mu \mathrm{m}$. 